\title{
Editorial: Los estudios de posgrados como eje primordial en la generación de investigación científica
}

Postgraduate studies as a primary axis in the generation of scientific research

\author{
Roberto N. Guerrero-Vega ${ }^{1}$ \\ (iD) https://orcid.org/0000-0003-3847-0326 \\ Universidad Politécnica de Nicaragua UPOLI
}

La investigación científica y los estudios de posgrados no son considerados un asunto tribal para las instituciones de educación superior, dado que, constituyen una gran parte de la razón de ser de las Universidades (Bifano, 2008). Según Guevara Pérez (2005) las instituciones de educación superior actúan como entes dinámicos que proporcionan cambios para el desarrollo de las sociedades, por ello, entre sus obligaciones primordiales deben de ofertar a sus maestros, colaboradores y egresados la oportunidad de alcanzar el perfeccionamiento de sus profesiones y conocimientos.

De acuerdo con Casimiro Urcos (2020) en los países en vías de desarrollo la educación posgraduada alcanza a ser, más que una inversión rentable, ya que, constituye un elemento estratégico para la creación del saber científico, técnico y humanístico; elementos que tanto requieren los miembros de una sociedad, todo ello partiendo de la investigación. Por estas razones los estudios de posgrados son referentes como los espacios más idóneos para aportar nuevos conocimientos al campo de la investigación científica.

Por tanto, las maestrías y doctorados son considerados estudios formativos, encaminados a la obtención de un grado académico, mismo que es alcanzado de manera legitima por medio de la investigación. El candidato a doctor deberá ser capaz de contribuir al acervo del conocimiento científico a través de la originalidad y difusión de su investigación (Espinoza Babilon, 2014).

\footnotetext{
${ }^{1}$ Doctor en Métodos Alternos de Solución de Conflictos, Universidad Autónoma de Nuevo León. Director del Centro de Mediación y Arbitraje de la Universidad Politécnica de Nicaragua y Editor general de la Revista Ciencia Jurídica y Política Email: derechorac@upoli.edu.ni
} 
En este sentido la Escuela de Ciencias Jurídicas y Políticas —ECJP_ quien es parte de la Universidad Politécnica de Nicaragua - UPOLI - desde su creación ha ofertado una variedad de maestrías que además de reforzar el enfoque profesionalízate de sus participantes en las diferentes áreas del derecho, también se ha concentrado en elevar el componente de la investigación científica, prueba de ello, es la reciente integración de módulos dirigidos a la investigación dentro de los programas de maestría, los cuales, tienen por objetivo contribuir con los proyectos de investigación que debe de entregar cada maestrante al culminar sus estudios de posgrado.

De igual forma también debo mencionar la existencia del primer Doctorado en Derecho ofertado por la ECJP, titulado "Nuevas tendencias del derecho en el siglo XXI", el cual es considerado eje primordial en la generación y divulgación de conocimiento científico. Cabe señal, que la divulgación de este conocimiento se realiza a través de una sinergia entre la Revista Ciencia Jurídica y Política y la primera edición del programa Doctoral, ambos productos de la ECJP.

En conclusión, la Universidad y los estudios de posgrados son cimientos esenciales para la generación de las investigaciones de índole académico y científico, por tal razón en esta ocasión la ECJP tiene el honor de presentar el número 13 de la "Revista Ciencia Jurídica y Política“, cuyo ejemplar está compuesto por un editorial, cuatro artículos científicos derivados de investigaciones concluidas o en curso y cuatro artículos de índole reflexivo. Los trabajos referidos tienen relación con las siguientes líneas temáticas: Los Métodos Alternos de Solución de Conflictos — MASC_- Relaciones Internacionales, Derechos Humanos — DD. HH.- - Derecho ambiental, penal y la criminología.

En el área de los MASC, contamos con un estudio cualitativo elaborado por los doctores Karla Sáenz, Elsa Zurita, Carlos Alejandro García Zúñiga de la Universidad Autónoma de Nuevo León - México, en donde nos presenta el avance de una investigación que se encuentra en curso titulada "Virtudes interpersonales del mediador en el proceso de mediación transformativa y su relación con la restauración de las partes intervinientes en el Estado de Nuevo León“. Este artículo sostiene como objetivo primordial reconocer la necesidad de desarrollar en los mediadores y mediadoras la virtud de la tolerancia para 
fomentar personas más abiertas a entender las controversias, por ello, también se analizan los antecedentes de la tolerancia como una virtud y su pertinencia dentro del proceso de mediación.

En cuanto a la línea de la Relaciones Internacionales, contamos con los valiosos aportes de las doctoras Gabriela Mata-Sánchez y Patricia Sepúlveda Chapa, quienes nos comparten su artículo "La legitimidad social de los organismos internacionales: el caso de la OMS en México durante la pandemia de COVID-19“ el cual es derivado de una investigación. En el referido artículo ambas doctoras plantean como eje primordial analizar la legitimidad social de la OMS en el contexto de la pandemia de COVID-19 en México, de lo cual se presentan resultados impactantes que devienen de diferentes sectores de la población en la cual se aplicó el instrumento de recolección.

Por otra parte, el Dr. en criminología Idrissa Sangaré comparte con nosotros su artículo reflexivo titulado "Hibridación del terrorismo y crimen organizado desde áfrica occidental hasta américa latina vs derechos humanos“. En este aporte el maestro desarrolla el impacto que tienen las organizaciones dedicadas al terrorismo con respecto a los nexos que desarrollan con el crimen organizado, en este sentido el autor hace mención de las rutas y países en los cuales este tipo de organizaciones fomentan actividades delictivas y delimita quienes son las personas vulnerables ante este actuar.

En el área del derecho público y social, encontramos la contribución de la Dra. Sandy Elizabeth Martínez Lara, quien nos comparte su artículo titulado “ Mecanismos del proceso de adaptación de alumnos extranjeros en la Universidad de Murcia, de España“ el cual es derivado de una investigación realizada en el año 2016. El objetivo primordial es establecer los factores asociados con el proceso de adaptación cultural en la Universidad de Murcia, por parte de los estudiantes de posgrado de la Facultad de Ciencias Políticas y Relaciones Internacionales que realizaron una movilidad académica.

Por su parte el candidato a doctor Geormar Vargas-Téllez nos comparte el articulo reflexivo titulado “Aproximación teórica a la prevención del delito y la seguridad pública”. En este articulo el autor plantea como objetivo primordial, identificar las finalidades de la 
prevención del delito vista desde la función disuasoria que realiza el derecho penal y el rol de la seguridad pública.

Dando continuidad a la línea del Derecho Penal, contamos con la participación del Dr. Marvin Ernesto Mora Rosales egresado del primer programa doctoral de derecho ofertado por la ECJP. En esta ocasión el maestro upoliano nos comparte, los resultados de la investigación doctoral titulada "Características de vulnerabilidad social del infractor adulto y su relación con la comisión de delitos graves en el departamento de Rivas. En referido trabajo el Dr. Mora mediante un análisis la situación de inseguridad de la ciudad de Rivas analiza el impacto de la criminalidad organizada y los factores ambientales que propiciaron el incremento delictivo, resaltando las características de vulnerabilidad social de los infractores de la ley y su relación con la comisión de delitos.

En lo que concierne a la línea de los Derechos Humanos, contamos con la valiosa participación de la Msc. Ivette Lenza García quien nos comparte su artículo de reflexión titulado “Asilo y Refugio. Un acercamiento desde el Derecho Internacional Humanitario“. Este trabajo aporta una conceptualización más amplia de lo que se debe de entender por asilo y refugiado, además esclarece cuales son los derechos que le asisten a las personas de acuerdo a la categorización de su condición migratoria.

Para concluir sobre la mención del contenido de este número 13, contamos con la destacada participación de la Dra. Yaelsy Lafita Cobas quien nos comparte su artículo de reflexión titulado “ Legislación ambiental y minera en Cuba. Aciertos y desaciertos”. En su contenido la autora realizar un análisis de las normas jurídicas existentes en Cuba que permiten la protección del medio ambiente ante los embates de la minería.

En conclusión, el fascículo 7(13) contiene artículos de alta calidad, en donde los autores desde sus diferentes temáticas colaboran con la difusión del conocimiento, crítico y científico. Por tanto, esperamos que este número sea de utilidad para la comunidad académica y para otros actores de la sociedad que se dedican a la investigación. 


\section{References.}

Bifano, C. (2008). Investigacion, posgrado y docencia de pregrado: Eje academico de la Universidad. En Autonomia Universitaria: Compromiso Academico y social (págs. 1-14). Caracas: CEA-UCV.

Casimiro Urcos, W. H. (2020). Los posgrados y las universidades peruanas. Mendive. Obtenido de http://mendive.upr.edu.cu/index.php/MendiveUPR/article/view/1876

Espinoza Babilon, J. R. (2014). Los estudios de posgrados y la investigacion. Acta Herediana, 54.

Guevara Perez, E. (2005). III Seminario sobre Educación de Postgrado. Postgrado de Especialización Pofesional bajo el. Merida, Venezuela: Universidad de Carabobo. 